\title{
Produção de biossurfactante por Chromobacterium violaceum ATCC 12472 utilizando milhocina e óleo de milho pós-fritura como nutrientes
}

\author{
Biosurfactant production by Chr omobacterium violaceum ATCC 12472 \\ using corn steep liquor and corn post-frying oil as nutrients
}

\author{
Adriana Almeida Antunes', Hélvia Waleuska Casullo de Araújo², Carlos Alberto Alves da Silva ${ }^{3}$, \\ Clarissa Daisy da Costa Albuquerque ${ }^{3}$, Galba Maria Campos-Takaki ${ }^{3 *}$
}

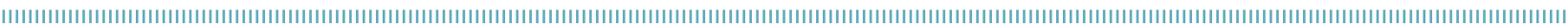

\begin{abstract}
RESUMO: $O$ interesse em surfactantes de origem microbiana tem aumentado consideravelmente nos últimos anos. A preocupaçáo ambiental entre os consumidores, combinada às novas legislaçóes de controle do meio ambiente, aumentou a procura por surfactantes naturais em substituição aos derivados petroquímicos. Os surfactantes microbianos têm sido testados em muitas aplicações ambientais e industriais, como na biorremediação, na dispersão de manchas oleosas e na recuperação de petróleo, substituindo os surfactantes químicos. Além disso, também podem ser utilizados nas indústrias de alimentos, cosméticos, detergentes e na agricultura. Neste trabalho foi produzido biossurfactante por Chromobacterium violaceum ATCC 12472, utilizando nutrientes de baixo valor agregado. A tensão superficial do biossurfactante produzido por C. violaceum ATCC 12472 teve valores entre 29 e $40 \mathrm{mN} / \mathrm{m}$. O melhor resultado foi observado no ensaio 8 (milhocina 8,0\%, lactose 1,0\% e óleo de milho pós-fritura 7,5\%) do planejamento fatorial, com 72 horas de fermentação, reduzindo a tensão superficial da água de 71 para $29 \mathrm{mN} / \mathrm{m}$. Os melhores resultados do índice $\left(\mathrm{E}_{24}\right)$ e da atividade de emulsificação (UEA) foram observados no ponto central, sendo utilizados para ambos os métodos o óleo de milho, que resultou no $\mathrm{E}_{24}$ de $2 \%$ e atividade com valores acima de 6 UAE. Os resultados obtidos demonstram o elevado potencial de C. violaceum na produçáo de um promissor biossurfactante.
\end{abstract}

PALAVRAS-CHAVE: Chromobacterium violaceum; biossurfactante; fontes alternativas.

\begin{abstract}
The interest in microbial surfactants has been increased considerably in the recent years. Environmental concern among consumers, combined with new laws control of the environment, the demand for natural surfactants, to replace petrochemicals. The microbial surfactants have been tested in many industrial and environmental applications, such as in bioremediation, oil dispersion and oil recovery, replacing chemical surfactants. Furthermore, they can also be used in the food, cosmetic, detergent and agriculture. However, increased environmental concern among consumers to new legislation combined control of the environment has led to demand for natural surfactants as alternatives to existing products. This work aimed to produce biosurfactant by Chromobacterium violaceum ATCC 12472, using low nutrient value. The surface tension of the biosurfactant produced by C. violaceum ATCC 12472 had values between 29 and $40 \mathrm{mN} / \mathrm{m}$. The best result was observed on the assay 8 (8.0\% of corn steep liquor, $1.0 \%$ of lactose and corn oil post-frying $7.5 \%)$ factorial design, within 72 hours of fermentation, lowering the surface tension of water from 71 to $29 \mathrm{mN} / \mathrm{m}$. The best results of the index $\left(\mathrm{E}_{24}\right)$ and emulsification activity (UEA) were observed in the central point, and using the both methods and corn post-frying oil resulted in $72 \%$ of emulsification, and the emulsification activity value of 6 UAE. The results demonstrate the high potential of C. violaceum promising in producing a biosurfactant.
\end{abstract}

KEYWORDS: Chromobacterium violaceum; biosurfactant; alternative sources.

\footnotetext{
'Programa de Pós-Graduação em Ciências Biológicas, Universidade Federal do Pernambuco (UFPE) - Recife (PE), Brasil. ${ }^{2}$ Departamento de Química, Universidade Estadual da Paraíba (UEPB) - Campina Grande (PB), Brasil .

${ }^{3}$ Núcleo de Pesquisas em Ciências Ambientais e Biotecnologia (NPCIAMB), Universidade Católica de Pernambuco (UNICAP) - Recife (PE), Brasil

*Autor correspondente: galba_takaki@yahoo.com.br

Recebido em: 21/03/2012. Aceito em: 17/06/2013
} 


\section{INTRODUÇÃO}

Os surfactantes microbianos constituem um grupo de moléculas estruturalmente diversificado que apresenta características anfipáticas, capazes de reduzir a atividade superficial de líquidos e a atividade interfacial entre líquidos, sólidos e gases, além de permitir a mistura e dispersão de substratos antes não solúveis em soluçôes aquosas (СAmeotra; MaKkAR, 2004; Nitschke; Pastore, 2002; Nitschke et al., 2005).

Estes biopolímeros podem ser classificados de acordo com a sua composição química e origem microbiana (Cameotra; Makkar, 2004; 2010; Mulligan, 2005). As principais classes dos biossurfactantes incluem: glicolipídeos, lipoproteínas, fosfolipídeos, lipídios neutros, ácidos graxos hidroxilados, glicolipídeos, lipopeptídeos e lipoproteínas (Lin, 1996).

Os biossurfactantes apresentam como uma de suas principais propriedades a redução da tensão superficial e interfacial entre líquidos, sólidos e gases e a capacidade de misturar e dispersar substratos antes insolúveis em soluçóes aquosas (Benincasa et al., 2004). Portanto, quanto menor a força de atração existente entre as moléculas do líquido, menor será a tensão superficial, proporcionando, assim, menor viscosidade e maior tendência à difusão (Cerqueira; Costa, 2009).

A grande maioria dos surfactantes disponíveis comercialmente é sintetizada a partir de derivados de petróleo. Entretanto, o aumento da preocupação ambiental entre os consumidores, combinado com novas legislaçôes de controle do meio ambiente, levou à procura por surfactantes naturais como alternativas aos produtos existentes (NiTsCHKE; Pastore, 2002).

Segundo a literatura, estes compostos oferecem diversas vantagens em relação aos surfactantes de origem química, tais como: baixa toxicidade e elevada biodegradabilidade; estabilidade em ampla faixa de $\mathrm{pH}$ e temperatura; e aceitabilidade ecológica, pois são potencialmente aplicáveis em processos de derramamentos de petróleo no mar, na limpeza de reservatórios e na biorremediaçáo de solos contaminados por substâncias hidrofóbicas, ou, ainda, em processos de recuperação melhorada do petróleo (MEOR). Vale destacar que eles podem ser produzidos a partir de fontes renováveis (Banat et al., 2000; Benincasa et al., 2002; MakKar; Cameotra, 2002; Rahman et al., 2002; Mulligan, 2005; Nitschke et al., 2005; Costa, 2005; Costa et al., 2006; BARRos et al., 2007; 2008).

Neste sentido, a produção de biossurfactantes a partir de substratos renováveis e de diferentes espécies microbianas permite além da possibilidade de variação de inúmeros parâmetros culturais, como tempo de cultivo, velocidade de agitaçâa, $\mathrm{pH}$ do meio e nutrientes. As moléculas obtidas apresentam características estruturais e propriedades físicas e biológicas distintas, o que os tornam comparáveis ou superiores aos surfactantes sintéticos. (BANAT et al., 2000; 2010; BenINCASA et al., 2002; MaKKAR;
Cameotra, 2002; Nitschke; Pastore, 2003; 2004a; 2004b; NitschKe et al., 2004; 2005; Mulligan, 2005; Costa et al., 2006; CAMEOTRA; MaKKaR, 2010). Estas propriedades tornam os surfactantes adequados para uma ampla gama de aplicaçóes industriais envolvendo detergência, emulsificação, lubrificação, capacidade espumante, molhante, solubilizante e dispersão de fases (BANat et al., 2000).

De acordo com Tuleva et al. (2002), os biopolímeros têm sido testados em muitas aplicaçôes ambientais, como na biorremediação, na dispersão de manchas oleosas e na recuperação melhorada de petróleo, substituindo os surfactantes químicos. Além disso, também podem ser utilizados nas indústrias de alimentos, cosméticos, detergentes e na agricultura.

A grande maioria dos surfactantes disponíveis comercialmente é sintetizada a partir de derivados de petróleo. Entretanto, o aumento da preocupaçáo ambiental entre os consumidores, combinado com novas legislaçóes de controle do meio ambiente, levou à procura por surfactantes naturais como alternativas aos produtos existentes (NitsChke; PAstore, 2002).

O sucesso da produção de biossurfactante depende do desenvolvimento de processos com o uso de matérias-primas de baixo custo, considerando que as mesmas são responsáveis por cerca de 30\% de todo o valor de produção. Apesar disso, poucos trabalhos têm sido publicados visando à produçáo de biossurfactantes a partir de resíduos, uma vez que a dificuldade é encontrar uma composição química adequada que permita o crescimento celular e a produção de metabólitos secundários de interesse (MAKKAR; Cameotra, 2002; Cameotra; MakKar, 2010).

Este trabalho teve como objetivo avaliar a produção de biossurfactante por Chromobacterium violaceum ATCC 12472 utilizando milhocina, lactose e óleo de milho pós-fritura como fontes de nitrogênio e carbono.

\section{MATERIAIS E MÉTODOS}

\section{Micro-organismo}

A linhagem de C. violaceum ATCC 12472 usada neste estudo encontra-se depositada no Banco de Culturas do Núcleo de Pesquisas em Ciências Ambientais da Universidade Católica de Pernambuco (UNICAP/PE), Brasil, sendo mantida no meio Agar Luria Bertani (LB), modificado (3 g/L triptona; $5 \mathrm{~g} / \mathrm{L}$ extrato de levedura; $5 \mathrm{~g} / \mathrm{L}$ cloreto de sódio; $5 \mathrm{~g} / \mathrm{L}$ de glicose e 17 $\mathrm{g} / \mathrm{L}$ de Agar) a $5^{\circ} \mathrm{C}$. A manutenção da cultura era realizada com repiques mensais para o meio $\mathrm{LB}$, crescida a temperatura de $30^{\circ} \mathrm{C}$ e, posteriormente, estocada à temperatura de $5^{\circ} \mathrm{C}$.

\section{Substratos}

Os substratos utilizados foram milhocina (resíduo gentilmente cedido pela indústria Corn Products de beneficiamento de 
milho), óleo de milho pós-fritura (cedido gentilmente pelo comércio informal - lanchonete) e lactose (Merck), sendo utilizados no meio de produção segundo as condiçóes estabelecidas no planejamento fatorial de $2^{3}$.

\section{Meio do pré-inóculo}

O pré-inóculo foi realizado transferindo-se a cultura de C. violaceum ATCC 12472 do meio sólido para $50 \mathrm{~mL}$ do caldo LB modificado ( $10 \mathrm{~g} / \mathrm{L}$ de triptona, $5 \mathrm{~g} / \mathrm{L}$ de extrato de levedura, $5 \mathrm{~g} / \mathrm{L}$ de cloreto de sódio e $5 \mathrm{~g} / \mathrm{L}$ de glicose), mantido à temperatura de $30^{\circ} \mathrm{C}$ por 12 horas a $150 \mathrm{rpm}$ até obtenção a $\mathrm{DO}_{600 \mathrm{~nm}}$ de 0,5 de absorbância, correspondendo a $10^{7} \mathrm{UFC} / \mathrm{mL}$.

\section{Planejamento fatorial}

Um planejamento fatorial completo de $2^{3}$, com 8 ensaios e 4 pontos centrais, foi realizado para analisar os efeitos principais e as interaçôes das variáveis independentes, concentraçôes de milhocina, lactose e óleo de milho pós-fritura, sob a variável resposta menor redução da tensão superficial do líquido metabólico livre de células. A Tabela 1 apresenta as variáveis utilizadas e os níveis estudados no referido planejamento, assim como a matriz descodificada.

Diagramas de Pareto com nível de significância de 95\% foram empregados para avaliar as estimativas dos efeitos principais lineares e de segunda ordem, em valor absoluto, dos fatores em relaçáo às variáveis respostas estudadas. No diagrama de Pareto, a magnitude de cada efeito foi representada pelas colunas, e a linha transversal às colunas correspondeu ao valor $\mathrm{p}=0,05$, indicando o efeito e o significado estatístico. As significâncias dos efeitos foram testadas por análise de variância (ANOVA), e as análises estatísticas dos planejamentos experimentais, incluindo os diagramas de Pareto, foram realizadas usando o programa Statistica ${ }^{\circledR}$ versão 8.0 (STATSOFt; 2001).

\section{Produção de biossurfactante por Chromobacterium violaceum ATCC 12472}

Para a produção de biossurfactante foram realizadas fermentaçôes utilizando 12 Erlenmeyers de $250 \mathrm{~mL}$ de capacidade

Tabela 1. Valores das variáveis independentes nos níveis -1 e +1 e do ponto central.

\begin{tabular}{lccc} 
& \multicolumn{3}{c}{ Níveis } \\
\cline { 2 - 4 } Variável independente & -1 & $\mathbf{0}$ & $\mathbf{+ 1}$ \\
Milhocina (\%) & 1,0 & 3,50 & 6,0 \\
\hline Lactose (\%) & 0,2 & 0,60 & 1,0 \\
\hline Óleo de milho pós-fritura (\%) & 5,0 & 6,25 & 7,5 \\
\hline
\end{tabular}

contendo $100 \mathrm{~mL}$ do meio de cultura com diferentes concentraçōes de lactose ( 0,2 a $1,0 \%)$, óleo de milho pós-fritura $(5,0$ a 7,5\%) e milhocina (1,0 a 6,0\%), adicionados de $3 \mathrm{~mL}$ do pré-inóculo com $\mathrm{DO}_{600 \mathrm{~nm}}$ de 0,5 . Os frascos foram mantidos sob agitação orbital de $150 \mathrm{rpm}$, a $30^{\circ} \mathrm{C}$, durante 72 horas. Após este período foi realizada centrifugação a $10.000 \mathrm{xg}, 5^{\circ} \mathrm{C}$, seguida de filtração em membrana Millipore de $0,22 \mathrm{~mm}$, a fim de separar as células do líquido metabólico. Nele mesmo foram realizadas as determinaçôes de tensão superficial, índice e atividade de emulsificação e $\mathrm{pH}$.

\section{Determinação do pH}

As determinaçóes do $\mathrm{pH}$ inicial e final foram realizadas em potenciômetro Orion (modelo 310) a partir do líquido metabólico livre de células.

\section{Determinação da tensão superficial}

A determinação da tensão superficial do líquido metabólico livre de células contendo o biossurfactante após 72 horas de cultivo foi medida com o uso de tensiômetro automático (modelo Sigma 70-KSV Ltd., Finland) utilizando a técnica do anel de DU NUOY, segundo metodologia descrita por KuYukina et al. (2001).

\section{Determinação do índice emulsificação}

Para a determinação do índice de emulsificação, as amostras retiradas após o processo fermentativo de 72 horas foram centrifugadas a $10.000 \mathrm{x}$ g durante 15 minutos e, em seguida, analisadas segundo a metodologia descrita por Cooper e Goldenberg (1987). Adicionaram-se em um tubo graduado 1,0 $\mathrm{mL}$ de óleo de milho e $2 \mathrm{~mL}$ do líquido metabólico livre de células, e a mistura foi agitada em vórtex por 2 minutos. $\mathrm{O}$ índice de emulsificação após 24 horas $\left(\mathrm{E}_{24}\right)$ foi calculado dividindo-se a altura da coluna de líquido emulsionado pela altura total do líquido no tubo, multiplicando-se por 100 para fornecer o percentual da emulsão formada.

\section{Determinação da atividade de emulsificação}

A determinação da atividade de emulsificaçáo do líquido metabólico livre de células foi realizada segundo a metodologia descrita por Cirigliano e Carman (1984). Foram colocados 2,0 $\mathrm{mL}$ do líquido metabólico livre de células, adicionado de 2,0 mL do tampão acetato de sódio $0,1 \mathrm{M}(\mathrm{pH} 3)$, com $1,0 \mathrm{~mL}$ de n-hexadecano ou óleo de milho em tubos graduados, e a mistura foi agitada em vórtex por 2 minutos. Após 10 minutos de repouso, as emulsôes formadas foram retiradas com o auxílio da pipeta Pasteur, colocadas em uma cubeta e, 
posteriormente, lidas em espectrofotômetro no comprimento de onda de $540 \mathrm{~nm}$. O resultado foi calculado em absorvância, expresso em UAE (unidade de atividade de emulsificação). Todos os testes foram realizados em duplicata.

Para a determinação do índice de emulsificação, as amostras retiradas após 72 horas de fermentação foram centrifugadas a $10.000 \mathrm{x} \mathrm{g}, 5^{\circ} \mathrm{C}$, durante 15 minutos, e filtradas em membrana da Millipore de 0,22 $\mathrm{mm}$. Em seguida, foram analisadas segundo a metodologia descrita por Cirigliano e CARMan (1984). Colocou-se 2,0 mL do líquido metabólico livre de células, adicionado a $2,0 \mathrm{~mL}$ do tampáo acetato de sódio $0,1 \mathrm{M}$ ( $\mathrm{pH}$ 3), com 1,0 mL de n-hexadecano ou óleo de milho em tubos graduados, e a mistura foi agitada em vórtex por 2 minutos. Após 10 minutos de repouso, as emulsóes formadas foram retiradas com o auxílio da pipeta de Pasteur, inseridas em uma cubeta e, posteriormente, lidas em espectrofotômetro no comprimento de onda a $540 \mathrm{~nm}$. O resultado foi calculado pela multiplicação do valor de absorbância por dois e representado por UAE. Os testes foram realizados em duplicata.

\section{RESULTADOS E DISCUSSÃO}

\section{Produção de biossurfactante por Chromobacterium violaceum ATCC \\ 12472}

De acordo com HaBA et al. (2000) e BAtista et al. (2006), os critérios utilizados para selecionar micro-organismos produtores de biossurfactantes são a habilidade em reduzir a tensão superficial abaixo de $40 \mathrm{mN} / \mathrm{m}$ e o índice de emulsificação da emulsão, mantendo-se pelo menos $50 \%$ do volume da emulsão original 24 horas depois da sua formação $\left(\mathrm{E}_{24}\right)$. A tensão superficial do isolado de $C$. violaceum ATCC 12472 determinada neste estudo alcançou valores entre 28 e $40 \mathrm{mN} / \mathrm{m}$. No entanto, o melhor resultado foi observado na condição 8 do planejamento fatorial, com concentraçôes de $6,00 \%$ de milhocina, 1,00\% de lactose e 7,50\% de óleo de milho, reduzindo a tensão da água de $71,00 \mathrm{mN} / \mathrm{m}$ para $28,98 \mathrm{mN} / \mathrm{m}$ em 72 horas de cultivo (Tabela 2 ).

O mesmo valor de 28,3 foi encontrado por Gouveia et al. (2003) em 96 horas de cultivo, utilizando Pseudomonas aeruginosa com o Meio Sais e Minerais (MSM) e adicionado de glicose. Contudo, os resultados obtidos foram inferiores aos encontrados por SiLva et al. (2009), com a utilização do micro-organismo Pseudomonas fluorescens em meio com petróleo, a menor tensão superficial foi 30,04 mN/m, em 60 horas de cultivo. Pirôllo et al. (2008), utilizando Pseudomonas aeruginosa, encontrou valores em torno de $33 \mathrm{mN} / \mathrm{m}$ quando cultivados em ensaios com $20 \%$ de querosene e $30 \%$ de óleo diesel. AnTUnes et al. (2006), com o mesmo isolado de C. violaceum ATCC 12472, obteve tensóes mais elevadas. Com o meio Luria Bertani suplementado com óleo de soja encontrou reduçáo da tensão superficial para $33,1 \mathrm{mN} / \mathrm{m}$; já com óleo de canola foi de $33,4 \mathrm{mN} / \mathrm{m}$; com óleo de milho foi de $34,21 \mathrm{mN} / \mathrm{m}$ e com glicose, 34,31 $\mathrm{mN} / \mathrm{m}$, todos com 72 horas de cultivo. Castro (2005), utilizando Pseudomonas aeruginosa em meio MLR mais glicerol, encontrou o valor de 44,6 mN/m em 96 horas de cultivo. Portanto, os resultados aqui descritos para C. violaceum ATCC 12472 foram superiores aos relatados na literatura sobre o tema, demonstrando que os substratos alternativos influenciaram de forma positiva a redução da tensão superficial e confirmando a produção de biossurfactante.

\section{Efeitos das concentrações de milhocina, lactose e óleo de milho sobre o bioprocesso}

No diagrama de Pareto de efeitos padronizados ilustrado na Figura 1, pode-se observar, para um nível de confiança de

Tabela 2. Matriz descodificada do planejamento fatorial de $2^{3}$ e os resultados da produção de biossurfactante por meio da redução da tensão superficial do líquido metabólico livre de células.

\begin{tabular}{lcccc} 
Ensaios & Milhocina (\%) & Lactose (\%) & Óleo de milho (\%) & Tensão superficial (mN/m) \\
\hline 1 & 1,0 & 0,2 & 5,00 & 31,04 \\
\hline 2 & 1,0 & 1,0 & 5,00 & 40,28 \\
\hline 3 & 6,0 & 0,2 & 5,00 & 35,71 \\
\hline 4 & 6,0 & 1,0 & 5,00 & 29,94 \\
\hline 5 & 1,0 & 0,2 & 7,50 & 34,79 \\
\hline 6 & 1,0 & 1,0 & 7,50 & 34,40 \\
\hline 8 & 6,0 & 0,2 & 7,50 & 33,45 \\
\hline 9 & 6,0 & 1,0 & 7,50 & 28,98 \\
\hline 10 & 3,5 & 0,6 & 6,25 & 30,77 \\
\hline 11 & 3,5 & 0,6 & 6,25 & 29,56 \\
\hline 12 & 3,5 & 0,6 & 6,25 & 30,46 \\
\hline
\end{tabular}


$95 \%$, que as concentraçôes em níveis mais elevados de milhocina $(6,0 \%)$, lactose $(1,0 \%)$ e óleo de milho pós-fritura $(7,5 \%)$ foram as que mais favoreceram a redução da tensão superficial do líquido metabólico livre de células. A interação entre as variáveis independentes lactose e óleo de milho pós-fritura e milhocina separadamente, nesta ordem, produziram efeitos negativos, favorecendo com significância estatística a redução da tensão superficial. Por outro lado, a interaçáo entre lactose e o óleo de milho pós-fritura, óleo de milho pós-fritura e lactose separadamente, e a interação da milhocina com óleo de milho pós- fritura, nesta ordem, também produziu efeitos negativos, mas sem significância do ponto de vista estatístico, na redução da tensão superficial (Fig. 1).

\section{Efeitos das concentrações de milhocina, lactose e óleo de milho pós-fritura sobre o bioprocesso}

De acordo com o diagrama de Pareto, ilustrado na Figura 1, verifica-se que a interaçáo da lactose com a milhocina, o aumento da concentração de milhocina, a interação da lactose com o óleo de milho e o aumento da concentração de óleo de milho exerceram efeitos negativos, estatisticamente significativos, sobre o crescimento da tensão superficial, ou seja, favoreceram a redução da tensão superficial. $\mathrm{O}$ aumento da concentração de lactose e a interaçáo da milhocina com o óleo de milho não contribuíram com significância estatística para a redução da tensão superficial.

Portanto, o diagrama de Pareto apresenta como efeitos padronizados, para um nível de confiança de $95 \%$, que as concentraçôes em níveis mais elevados de milhocina (6\%), lactose

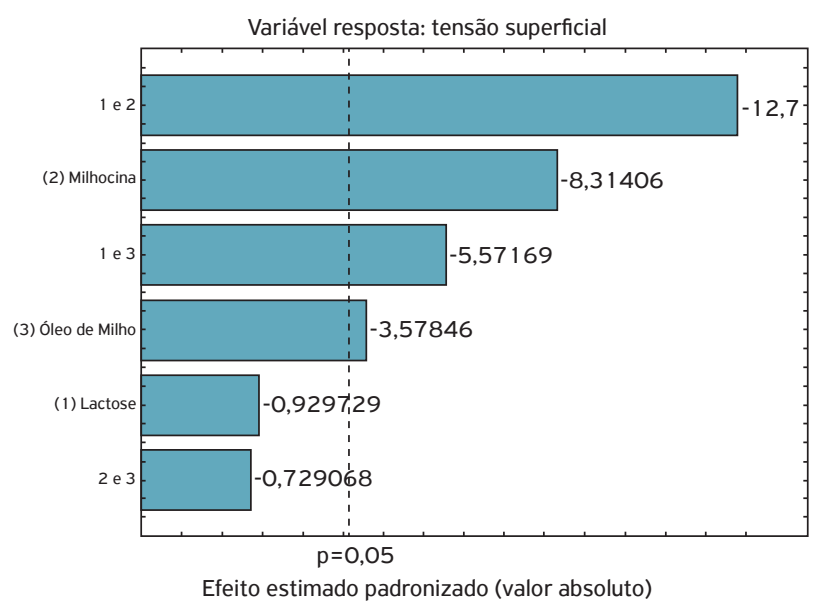

Figura 1. Diagrama de Pareto para planejamento fatorial $2^{3}$ tendo como variáveis independentes (1) lactose, (2) milhocina e (3) óleo de milho, e como variável resposta a tensão superficial. $O$ ponto no qual os efeitos estimados são estatisticamente significativos ( $p=0,05)$ está indicado pela linha vertical vermelha tracejada.
(1\%) e óleo de milho $(7,5 \%)$ foram as que mais favoreceram a redução da tensão superficial do líquido metabólico livre de células. Por outro lado, a interaçáo entre lactose e o óleo de milho, óleo de milho separadamente, lactose separadamente, e a interação da milhocina com óleo de milho, nesta ordem, também produziram efeitos negativos, mas sem significância do ponto de vista estatístico, na redução da tensão superficial.

\section{Propriedade emulsificante do biossurfactante produzido por Chromobacterium violaceum}

Um importante parâmetro de avaliação do poder emulsificante é o índice de Emulsificação $\left(\mathrm{E}_{24}\right)$ e a estabilidade da emulsão (Cooper; Goldennerg, 1987; Abu-Ruwaida et al., 1991; Costa, 2005; Nitschke e Pastore, 2006). O melhor resultado do índice de emulsificação foi determinado pelos pontos centrais do planejamento fatorial, obtendo-se em torno de $72 \%$ de emulsificação com o óleo de milho no período de 72 horas (Fig. 2).

RochA et al. (2006; 2007) obtiveram resultados semelhantes de $71,79 \%$ de emulsificação para óleo de soja e inferiores de $16,50 \%$ de querosene, respectivamente, utilizando Pseudomonas aeruginosa em meio de suco de caju; enquanto Bezerra et al. (2012) encontraram valores em torno de 64,29\% utilizando querosene comercial com o mesmo micro-organismo. BARBOSA e PAZ (2007) utilizaram a C. violaceum e encontraram valores inferiores $(43,30 \%)$ de emulsificaçáo no período de 60 horas após 2 minutos utilizando óleo de pequi. Contudo, após 24 horas de repouso, o valor foi reduzido para $8,33 \%$.

A atividade de emulsificação mostrou excelentes resultados com o óleo de milho para o biossurfactante produzido por C. violaceum, obtendo-se valores acima de $6 \mathrm{UAE}$ para as amostras provenientes de todas as condiçóes descritas no planejamento fatorial. $\mathrm{O}$ n-hexadecano também formou emulsão em todas as condiçōes, variando entre 0,9 a valores acima de $6 \mathrm{UAE}$ (Fig. 3). A literatura descreve valores máximos de 0,72 UAE em

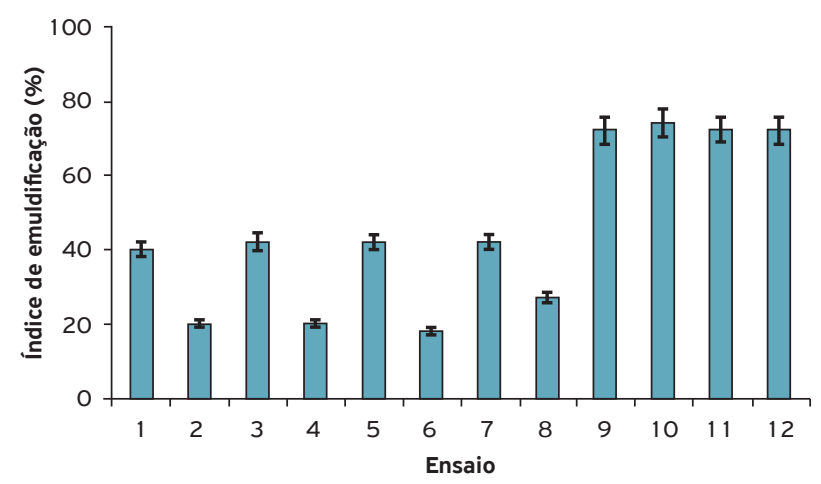

Figura 2. Resultados do índice de emulsificação $\left(E_{24}\right)$ do biossurfactante produzido por Chormobacterium violaceum ATCC 12472 nos ensaios do planejamento fatorial $2^{3}$ usando óleo de milho como fase oleosa (Tabela 1 ). 


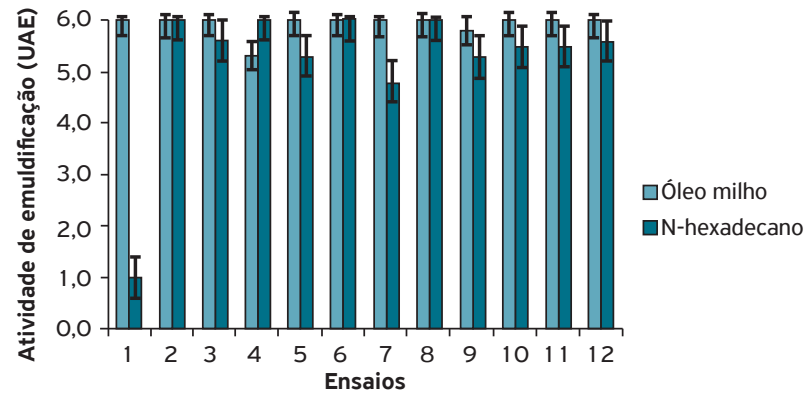

Figura 3. Atividade emulsificante do biossurfactante produzido por Chromobacterium violaceum ATCC 12472 nos ensaios do planejamento fatorial $2^{3}$ utilizando óleo de milho e n-hexadecano.

n-hexadecano para o biossurfactante produzido em meio contendo manitol por Bacillus subtilis (Bueno, 2008), e PAZ et al. (2006) encontraram valores máximos de 5,90 UAE utilizando Geobacillus stearothermophilus com n-hexadecano em 96 horas.

\section{CONCLUSÕES}

O uso de um planejamento experimental demonstrou ser uma ferramenta importante e aplicável para evidenciar a produção de biossurfactante por $C$. violaceum ATCC 12472, assim como para avaliar o comportamento das variáveis independentes sobre a reduçáo da tensão superficial, atividade e índice de emulsificação, apresentando influência dos parâmetros independentes do processo. As propriedades redução da tensão superficial e capacidade emulsificante indicam perspectivas futuras para a aplicação do biossurfactante em processos de biorremediação de áreas contaminadas, além de demonstrar o potencial deste organismo como um candidato para a remediação de óleo bruto e recuperaçáo melhorada do petróleo.

\section{AGRADECIMENTOS}

Os autores agradecem aos órgãos de fomento Conselho Nacional de Desenvolvimento Científico e Tecnológico (CNPq), Coordenação de Pessoal de Aperfeiçoamento de Nível Superior (CAPES) e Fundação de Amparo à Pesquisa e Tecnologia do Estado de Pernambuco (FACEPE) e à Universidade Católica de Pernambuco (UNICAP), em especial ao Núcleo de Pesquisas em Ciências Ambientais e Biotecnologia (NPCIAMB), pelo uso das instalaçóes.

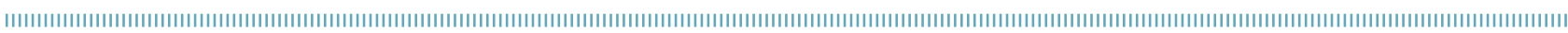

\section{REFERÊNCIAS}

ABU-RUWAIDA, A.S.; MAACHI, R.; AMRANE, A.; BOUDERGUA, A.N. Isolation of biosurfactant-producing bacteria product characterization, and deviation. Acta Biotechnologica, v.11, n.4, p.315-324, 1991.

ANTUNES, A.A.; SILVA, M.L.R.B.; SILVA, C.A.A.; CAMPOSTAKAKI, G.M. Characterization of Chromobacterium violaceum isolated from Paca River, Pernambuco, Brazil. Revista de Biologia e Ciências da Terra, n. 1, p.48-55, 2006.

BANAT, I.M.; MAKKAR, R.S.; CAMEOTRA, S.S. Potential commercial applications of microbial surfactants. Applied Microbiology and Biotechnology, v.53, n.5, p.495-508, 2000.

BANAT, I.M.; FRANZETTI, A.; GANDOLFI, I.; BESTETTI, G.; MARTINOTTI, M.G.; FRACCHIA, L.; SMYTH, T.J.; MARCHANT, R. Microbial biosurfactants production, applications and future potential. Applied Microbiology and Biotechnology, v.87, n.2, p.427-444, 2010.

BARBOSA, M.M.C.; PAZ, M.C.F. Produção de biossurfactantes por Chromobacterium violaceum utilizando como substrato óleo vegetal (óleo de pequi). In: CONGRESSO DE PESQUISA E INOVAÇÃO DA REDE NORTE NORDESTE DE EDUCAÇÃO TECNOLÓGICA, 2., 2007, João Pessoa, PB. Resumos. João Pessoa: 2007.
BARROS, F.F.C.; QUADROS, C.P.; MARÓSTICA JR, M.R.; PASTORE, G.M. Surfactina: propriedades químicas, tecnológicas e funcionais para aplicações em alimentos. Química Nova, v.30, n.2, p.409-414, 2007.

BARROS, F.F.; PONEZI, A.N.; PASTORE, G.M. Production of biosurfactant by Bacillus subtilis LB5a on a pilot scale using cassava wastewater as substrate. Journal of Industrial Microbiology \& Biotechnology, v.35, n.9, p.1071-1078, 2008.

BATISTA, S.B.; MOUNTEER, A.H.; AMORIM, F.R.; TÓTOLA, M.R. Isolation and characterization of biosurfactant/bioemulsifierproducing bacteria from petroleum contaminated sites. Bioresource Technology, v.97, n.6, p.868-875, 2006.

BENINCASA, M.; CONTIERO, J.; MANRESA, M.A., MORAES, I.O. Rhamnolipid production by Pseudomonas aeruginosa LBI growing on soapstock as the sole carbon source. Journal of Food Engineering, v.54, n.4, p.283-288, 2002.

BENINCASA, M.; ABALOS, A.; OLIVEIRA, I.; MANRESA, A. Chemical structure, surface properties and biological activities of the biosurfactant produced by Pseudomonas aeruginosa LBI from soapstock. Antonie Van Leeuwenhoek, v.85, n. 1, p.1-8, 2004. 
BEZERRA, M.S.; HOLANDA, V.C.D.; AMORIM, J.A.; MACEDO, G.R.; SANTOS, E.S. Produção de biotensoativo utilizando Pseudomonas aeruginosa (P.A.) e resíduo agroindustrial (manipueira) como substrato. Holos, v.1, p.14-27, 2012.

BUENO, S.M. Bactérias produtoras de biossurfactantes: isolamento, produção, caracterização e comportamento num sistema modelo. 2008. 89p. Tese (Doutorado - Engenharia, Ciência e Tecnologia de Alimentos) - Instituto de Biociências, Letras e Ciências Exatas, Universidade Estadual Paulista "Júlio de Mesquita Filho", São José do Rio Preto, 2008.

CAMEOTRA, S.S.; MAKKAR, R.S. Recent applications of biosurfactants as biological and immunological molecules. Current Opinion in Microbiology, v.7, n.3, p.262-266, 2004.

CAMEOTRA S.S.; MAKKAR, R.S. Biosurfactant-enhanced bioremediation of hydrophobic pollutants. Pure Applied Chemistry, v.82, n.1, p.97-116, 2010.

CASTRO, C.J.T.L. Avaliação da produção de raminolipidios por bactérias isoladas de poços de petróleo. 2005. 53p. Dissertação (Mestrado - Biotecnologia) - Universidade Federal de Pernambuco, Recife, 2005.

CERQUEIRA, V.S.; COSTA, J.A.V. Biodegradação de tolueno e óleo de pescado em solos impactados utilizando surfactantes químico e biológico. Química Nova, v.32, n.2, p.395-400,2009.

CIRIGLIANO, M.C.; CARMAN, G.M. Isolation of a bioemulsifier from Candida lipolytica. Applied and Environmental Microbiology, v.48, n.4, p.747-750, 1984.

COOPER, D.G.; GOLDENBERG, B.G. Surface-active agents from two Bacillus species. Applied and Environmental Microbiology, v.53, n.2, p. 224-229, 1987.

COSTA, G.A.N. Produção biotecnológica de surfactante de Bacillus subtilis em resíduo agroindustrial, caracterização e aplicações. 2005. 87p. Dissertação (Mestrado - Ciência de Alimentos) Faculdade de Engenharia de Alimentos, Universidade Estadual de Campinas, Campinas, 2005.

COSTA, S.G.V.A.O.; NITSCHKE, M.; HADDAD, R.; EBERLIN, M.N.; CONTIERO, J. Production of Pseudomonas aeruginosa LB1 rhamnolipids following growth on Brazilian native oils, Process Biochemistry, v.41, p.483-488, 2006.

GOUVEIA, E.R.; LIMA, D.P.A.; DUARTE, M.S.; LIMA, G.M.S.; ARAÚJO, J.M. Produção de biossurfactantes por bactérias isoladas de poços de petróleo. Revista Biotecnologia Ciência e Desenvolvimento, n.30, p.39-45, 2003.

HABA E.; ESPUNY, M.J.; BUSQUETS, M.; MANRESA, A. Screening and production of rhamnolipids Pseudomonas aeruginosa 47T2 NCIB 40044 from waste flying oils. Journal Applied Microbiology, v.88, p.379-387, 2000.

KUYUKINA, M.S.; IVSHINA, I.B.; PHILP, J.C.; CHRISTOFI, N.; DUNBAR, S.A.; RITCHKOVA, M.I. Recovery of Rhodococcus biosurfactants using methyl tertiary-butyl ether extraction. Journal of Microbiological Methods, n.46, n.2, p.149-156, 2001.
LIN, S.C. Biosurfactants; recent advances. Journal of Chemical Technology and Biotechnology, v.66, n.2, p.109-120, 1996.

MAKKAR, R.S.; CAMEOTRA, S.S. An update on the use of unconventional substrates for biosurfactant production and their new applications. Applied Microbiology and Biotechnology, v.58, n.4, p.428-34, 2002.

MULLIGAN, C.N. Environmental applications for biosurfactants. Environmental Pollution, v.133, n.2, p.183-198, 2005.

NITSCHKE, M.; PASTORE, G.M. Biossurfactantes: propriedades e aplicações. Química Nova, v.25, n.5, p.772-776, 2002.

NITSCHKE, M.; PASTORE, G.M. Cassava flour wastewater as a substrate for biosurfactant production. Applied Biochemistry and Biotechnology, v.105-108, p.295-301. 2003.

NITSCHKE, M.; PASTORE, G.M. Biosurfactant production by Bacillus subtilis using cassava-processing effluent. Applied Biochemistry and Biotechnology, v.112, n.3, p.163-172, 2004a.

NITSCHKE, M.; PASTORE, G.M. Structural characterization and biological properties of a lipopeptide surfactant produced by Bacillus subtilis in cassava wastewater medium. Food Science (2) Biotechnology, v.13, n.5, p.591-596, 2004b.

NITSCHKE, M.; FERRAZ, C.; PASTORE, G.M. Selection of microrganisms for biosurfactant production using agroindustrial wastes. Brazilian Journal of Microbiology, v.35, p.81-85, 2004.

NITSCHKE, M.; COSTA, S.G.; CONTIERO, J. Rhamnolipid surfactants: an update on the general aspects of these remarkable biomolecules. Biotechnology Progress, v.21, n.6, p.1593-1600, 2005.

NITSCHKE, M.; PASTORE, G.M. Production and properties of a surfactant obtained from Bacillus subtilis grown on cassava wastewater. Bioresource Technology, v.97, n.2, p.336-341, 2006.

PAZ, M.C.F.; CEBALLOS, B.S.O.; ALBUQUERQUE, C.D.C.; CAMPOS-TAKAKI, G.M. Influência de cloreto de sódio e de cobre na produção de biomassa e biosurfactante por uma nova amostra de Geobacillus stearothermophilus UCP 986. Revista de Biologia e Ciências da Terra, n. 1, p.56-64, 2006.

PIRÔLLO M.P.; MARIANO, A.P.; LOVAGLIO, R.B.; COSTA, S.G.; WALTER, V.; HAUSMANN, R.; CONTIERO, J. Biosurfactant synthesis by Pseudomonas aeruginosa LBI isolated from a hydrocarbon-contaminated site. Journal of Applied Microbiology, v.105, n.5, p.1484-1490, 2008.

RAHMAN, K.S.; RAHMAN, T.J.; MCCLEAN, S.; MARCHANT, R.; BANAT, I.M. Rhamnolipid biosurfactant production by strains of Pseudomonas aeruginosa using low-cost raw materials. Biotechnology Progress, v. 18, n.6, p.1277-1281, 2002.

ROCHA, M.V.P.; OLIVEIRA, A.H.S.; SOUZA M.C.M.; GONÇALVES, L.R.B. Natural cashew apple juice as fermentation medium for biosurfactant production by Acinetobacter calcoaceticus. 
World Journal of Microbiology and Biotechnology, v.22, p.1295-1299, 2006.

ROCHA, M.V.P.; MENDES, J.S.; GIRO, M.E.A.; GONÇALVES, L.R.B. Estudo da estabilidade do biossurfactante produzido por Pseudomonas aeruginosa em suco de caju. In: PDPETRO, 4. 2007, Campinas, SP. Resumos. Campinas: 2007. p.1-10.

SILVA, T.A.L.; ARAÚJO, H.W.C.; TAMBOURGI, E.B.; SILVA, C.A.A.; TAKAKI, G.M.C. Potencial biotecnológico de uma nova linhagem de Pseudomonas fluorescens na produção de biossurfactante utilizando petróleo como substrato. Exacta, v.7, n. 1, p.31-37, 2009.

STATSOFT. Statistica (data analysis software system), version 8.0. 2001. Disponível em: <http://www.statsoft.com>. Acesso em: 21 mar. 2009.

TULEVA, B.K.; IVANOV, G.R.; CHRISTOVA, N.E. Biosurfactant production by a new Pseudomonas putida strain. Zeitschrift für Naturforschung, v.57, n.3-4, p.356-360, 2002. 